\title{
Utilização de Lactobacillus acidophilus e de acidificação direta na fabricação de queijo de minas frescal
}

\author{
[Lactobacillus acidophilus and of direct acidification to minas \\ frescal cheese production] \\ C.C.C. Alves ${ }^{1}$, N.D.H. Gemal ${ }^{2}$, M.A.S. Cortez $^{3}$, R.M. Franco ${ }^{3}$, S.B. Mano ${ }^{3}$ \\ ${ }^{1}$ Médica veterinária autônoma \\ ${ }^{2}$ Aluna de pós-graduação - Faculdade de Veterinária - UFF - Niterói, RJ \\ ${ }^{3}$ Faculdade de Veterinária - UFF - Niterói, RJ
}

\begin{abstract}
RESUMO
Três procedimentos foram adotados na elaboração de queijo de minas frescal: fabricação tradicional, com adição de ácido lático e com Lactobacillus acidophilus. As amostras dos queijos foram analisadas quanto à composição e, durante o período de estocagem de 30 dias, quanto à acidez - pH e acidez titulável - e contagem de L. acidophilus. A análise sensorial foi realizada por métodos sensoriais afetivos. A composição dos queijos nos diferentes procedimentos apresentou-se de acordo com os padrões esperados, com exceção do teor de gordura, que foi maior no queijo com adição de ácido lático. Durante a armazenagem, ocorreu aumento da acidez titulável em todos os procedimentos. A contagem de $L$. acidophilus foi acima de $10^{8} \mathrm{UFC} / \mathrm{g}$, caracterizando populações suficientes para classificar o queijo como alimento probiótico. $\mathrm{Na}$ análise sensorial, o de queijo de minas com a adição do probiótico foi o preferido pelos julgadores. O queijo de minas frescal foi apropriado para incorporação do probiótico, e o uso de $L$. acidophilus melhorou a qualidade sensorial e não alterou os parâmetros físico-químicos.
\end{abstract}

Palavras-chave: queijo, probióticos, Lactobacillus acidophilus, ácido lático

\begin{abstract}
Minas frescal cheese was produced in three different procedures: traditional manufacturing; with the addition of lactic acid; and with Lactobacillus acidophilus. Cheeses samples were analyzed for composition, and during the stock period of 30 days for acidity and $\mathrm{L}$. acidophilus count. The sensory analysis was carried out through sensory affective methods. The composition of cheeses in different treatments presented results in accordance to the standards expected for the product, except for fat content, which was higher in cheese with the addition of lactic acid. During the stock period, an increase of titulable acidity in all treatments was observed. The L. acidophilus count was above $10^{8} \mathrm{UFC} / \mathrm{g}$, characterizing sufficient population to classify the cheese as a probiotic food. At the sensory analysis, it was attested that the cheese which had the addition of probiotic was preferred among the tasters. The Minas Frescal cheese was appropriate for incorporation of probiotic and the use of L. acidophilus in cheese improved the sensory quality and did not alter the physicochemical parameters.
\end{abstract}

Keywords: cheese, probiotics, Lactobacillus acidophilus, lactic acid

\section{INTRODUÇÃO}

A produção de queijos representa uma das mais importantes atividades para os estabelecimentos industriais, pois $34 \%$ de todo o leite recebido é direcionado à fabricação de queijos
(Estatística..., 2010). Destaca-se o queijo de minas frescal, que apresenta ampla aceitação no mercado, excelente rendimento na fabricação e facilidade de produção. No entanto, devido ao alto teor de umidade, elevado valor de $\mathrm{pH}$ e grande manipulação durante a fabricação, é

Recebido em 2 de julho de 2010

Aceito em 5 de setembro de 2011

E-mail: claracalil@yahoo.com.br 
muito suscetível a alterações bioquímicas e microbiológicas que afetam as características de qualidade e durabilidade. Além disso, com frequência apresenta contaminação por diversos patógenos de importância para a saúde coletiva (Furtado e Lourenço Neto, 1994; Pinto et al., 2004).

No setor láteo, os alimentos funcionais, como os adicionados de microrganismos probióticos, apresentam elevado valor comercial, e muitos estabelecimentos industriais desenvolvem produtos tendo a promoção da saúde como relevante estratégia de propaganda. O Lactobacillus acidophilus é considerado um microrganismo probiótico em razão dos benefícios relacionados ao consumo (Garcia et $a l ., 2006)$, sendo muito utilizado na indústria de laticínios, na fabricação de diversos produtos. A acidificação de queijos utilizando ácido lático é descrita como uma forma de melhorar as características de produção e de preservação, pelo fato de diminuir as alterações qualitativas ao longo do tempo de estocagem (Fox, 2000).

Os objetivos do presente experimento foram obter as características físico-químicas do queijo de minas frescal produzido pelo procedimento tradicional, com a adição de probiótico e com a adição de ácido lático, determinar o pH e a acidez titulável durante o período de estocagem de 30 dias, efetuar a contagem de L. acidophilus durante o período de armazenagem e realizar a avaliação sensorial por meio de testes afetivos.

\section{MATERIAL E MÉTODOS}

Os queijos foram elaborados com leite previamente pasteurizado, utilizando-se quantidades de cloreto de cálcio (solução 50\%, Cap-Lab), coagulante microbiano comercial (produzido por cepa de Aspergillus niger var. awamori, marca Chr. Hansen) e cloreto de sódio (refinado extraiodado, marca Cisne), como o preconizado por Furtado e Lourenço Neto (1994). Para tal, adotaram-se três procedimentos na elaboração dos queijos: queijo de minas frescal tradicional (controle, T1); queijo de minas frescal adicionado de L.acidophillus (T2) e queijo de minas frescal adicionado de ácido lático (T3).
Para a produção do queijo de minas frescal com probiótico, foi utilizada a cultura comercial liofilizada de L. acidophilus (LA-5, fornecida pela Chr. Hansen), mantida sob congelamento a $18^{\circ} \mathrm{C}$ até o momento de uso. A concentração desejada para a inoculação direta no leite foi de $10^{8} \mathrm{UFC} / \mathrm{mL}$, obtida conforme indicação do fabricante (um envelope de $25 \mathrm{~g}$ para cada $500 \mathrm{~L}$ de leite). Na acidificação direta do leite com ácido lático, foram adicionados $2,5 \mathrm{~mL}$ de ácido lático para cada 10L de leite, previamente diluído em $25 \mathrm{~mL}$ de água destilada.

Para caracterizar o produto, foram realizadas análises para determinação de gordura, proteína, cinzas, acidez titulável, pH (Brasil, 2006), cloretos (Métodos, 1981) e umidade (Métodos, 2005). As análises das amostras de cada procedimento foram realizadas em duplicata, no primeiro dia após a produção, usando-se estatística descritiva simples. $\mathrm{Na}$ verificação das alterações de acidificação durante o armazenamento, foram realizadas análises de acidez titulável e pH (Brasil, 2006), em sete momentos, nos dias um, três, seis, 12, 18, 24 e 30 após a fabricação.

A contagem de L. acidophilus foi realizada no tratamento com probiótico, durante o período de armazenagem, nos mesmos sete momentos. O método utilizado foi o do inóculo em profundidade, com incubação a $35-37^{\circ} \mathrm{C}$, por 72 horas, em anaerobiose (Barreto et al., 2003; Garcia et al., 2006).

Os métodos sensoriais utilizados foram o método sensorial afetivo, o de aceitação segundo a escala de nove pontos e o de aceitação segundo a escala de atitude de nove pontos. Em cada teste, foram utilizados 44 julgadores, recrutados aleatoriamente. Os queijos foram analisados no sétimo dia de fabricação. Os resultados foram submetidos à análise de variância e à comparação de médias pelo teste de Tukey.

\section{RESULTADOS E DISCUSSÃO}

Os resultados referentes à composição porcentagem de gordura, proteína, cloretos, umidade e cinzas - das amostras de queijos, realizados no primeiro dia após a fabricação, encontram-se na Tab. 1. 
Utilização de Lactobacillus acidophilus...

Tabela 1. Composição e gordura no extrato seco (GES) dos queijos, segundo os procedimentos de elaboração

\begin{tabular}{ccccccc}
\hline \multirow{2}{*}{ Tratamento } & \multicolumn{5}{c}{ Composição } \\
\cline { 2 - 7 } & Umidade (\%) & Cinzas (\%) & Cloretos $(\%)$ & Proteína (\%) & $\begin{array}{c}\text { Gordura } \\
(\%)\end{array}$ & $\begin{array}{c}\text { GES } \\
(\%)\end{array}$ \\
\hline T1 & 64,61 & 4,07 & 1,90 & 16,74 & 18,88 & 53,35 \\
T2 & 63,43 & 4,26 & 1,72 & 15,63 & 18,88 & 51,63 \\
T3 & 60,74 & 5,08 & 2,05 & 17,30 & 24,55 & 62,53 \\
Média & 62,93 & 4,47 & 1,89 & 16,56 & 20,77 & 55,84 \\
DP & 1,983 & 0,536 & 0,165 & 0,849 & 3,273 & 5,860 \\
CV & 0,0315 & 0,120 & 0,087 & 0,0513 & 0,1576 & 0,1049 \\
\hline T1: & queijo de minas frescal tradicional (controle); T2: queijo de minas frescal adicionado de Lactobacillus
\end{tabular}
acidophilus; T3: queijo de minas frescal adicionado de ácido lático.

Os queijos apresentaram, em média, 55,8\% de gordura no extrato seco. Na legislação em vigor (Brasil, 1996), o queijo de minas frescal é classificado como semigordo - conteúdo de gordura no extrato seco entre $25 \%$ e $44,9 \%$. No presente experimento, em T1 e T2 obtiveram-se queijos gordos - gordura no extrato seco entre $45,0 \%$ e $59,9 \%$ - e em T3 queijo extragordo gordura no extrato seco de, no mínimo, $60 \%$. Isto provavelmente ocorreu em virtude do maior teor de gordura do leite utilizado como matériaprima, pois não houve padronização do teor de gordura do leite. Furtado e Lourenço Neto (1994) relataram que, na fabricação industrial, é comum a padronização para 3,0\% a 3,2\% de gordura.

A maior porcentagem de gordura também está relacionada ao tratamento com ácido lático, como observado em T3. Van Dender et al. (1999), ao estudarem o queijo de minas frescal, em diferentes metodologias de fabricação, encontraram resultados semelhantes aos do atual experimento, pois obtiveram valores mais altos de gordura nos queijos fabricados com ácido lático.

Quanto à composição, os resultados deste trabalho foram semelhantes aos de Lourenço Neto (1998) e Campos e Viotto (1999). O primeiro autor obteve os seguintes valores em queijos fabricados com ácido lático: $61,7 \%$ de umidade, $18,6 \%$ de gordura, $0,99 \%$ de cloretos e $16,3 \%$ de proteínas. Campos e Viotto (1999), ao estudarem o rendimento do queijo fabricado com o mesmo procedimento, encontraram $21,7 \%$ de gordura, $1,2 \%$ de cloretos e $16,0 \%$ de proteínas.

Neste trabalho, os valores de cloretos e gordura no procedimento tradicional foram semelhantes aos de Furtado e Lourenço Neto (1994) e Perry (2004). Furtado e Lourenço Neto (1994) encontraram os seguintes valores para o queijo de minas frescal: umidade entre $55 \%$ e $58 \%$, gordura entre $17 \%$ e $19 \%$ e cloretos entre $1,4 \%$ e $1,6 \%$. Perry (2004) sugeriu que os queijos contenham entre $0,5-2,0 \%$ de cloretos. Os teores de umidade encontrados estavam em conformidade com a legislação em vigor. $\mathrm{O}$ teor de umidade para o queijo de minas frescal, de acordo com o estipulado na legislação, é de no mínimo, $55 \%$, sendo o queijo classificado como de muito alta umidade (Brasil, 1996).

A composição nas amostras dos queijos com adição de L. acidophilus foi semelhante à do queijo tradicional, em todos os parâmetros estudados, o que indica que o probiótico usado não alterou a composição do queijo. Resultados semelhantes foram encontrados por Buriti et al. (2005) e Souza (2006). Buriti et al. (2005) constataram que não ocorreram diferenças significativas no rendimento, umidade, gordura e proteína nos queijos com adição de $L$. acidophilus, quando comparados ao tradicional. Souza (2006), ao avaliar a composição centesimal do queijo de minas frescal com adição do mesmo probiótico, obteve para o tratamento com L. acidophilus e para o tratamento tradicional, respectivamente, $65,6 \%$ e $66,3 \%$ de umidade, $16,2 \%$ e $15,7 \%$ de gordura, $10,0 \%$ e $12,9 \%$ de proteínas, $2,4 \%$ e $2,3 \%$ de cinzas.

Quanto à acidez titulável durante a armazenagem (Fig. 1), não houve diferença entre os tratamentos na acidez final dos queijos, principalmente entre a acidez do T2 e do T1. No T3 obteve-se a maior acidez titulável, o que também foi encontrado por Van Dender et al. (1999), que analisaram o queijo de minas frescal, adicionado de ácido lático e na fabricação tradicional, e verificaram que a acidez titulável do produto com ácido lático foi maior. 


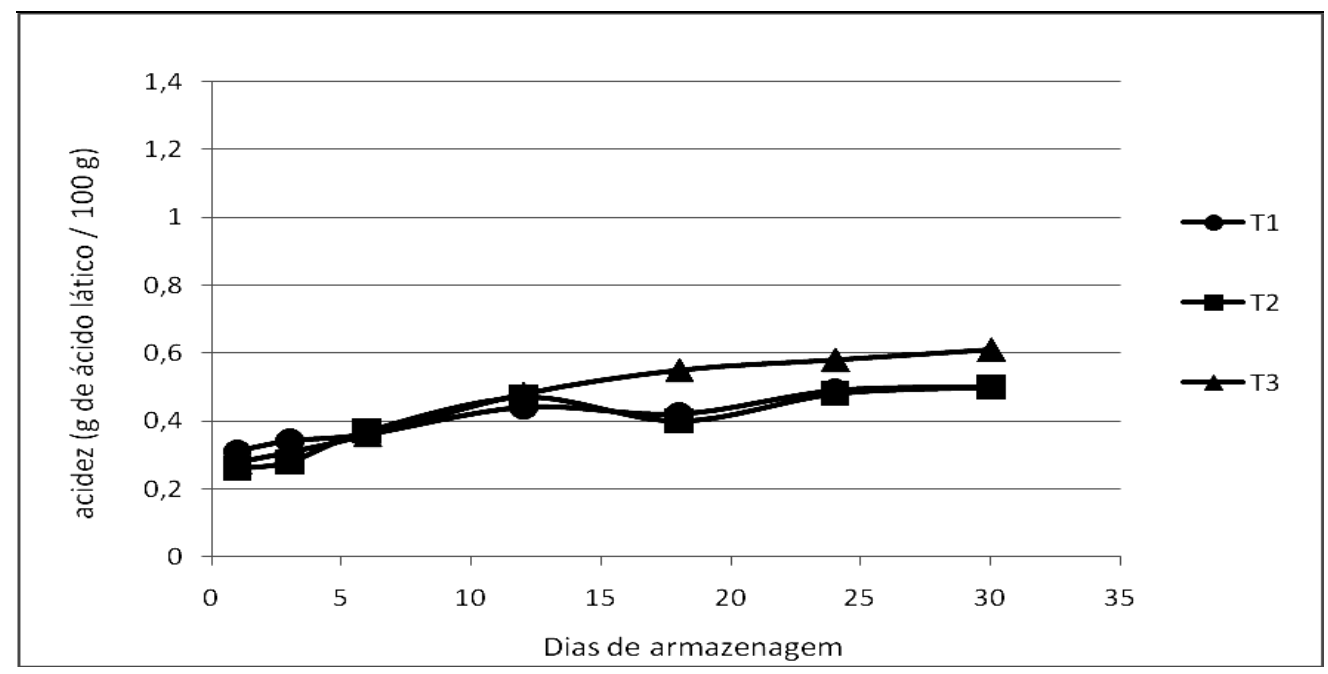

Figura 1. Acidez titulável, em gramas de ácido lático/100 gramas, ao longo do tempo de estocagem dos queijos, segundo os procedimentos de elaboração. T1: queijo de minas frescal tradicional (controle); T2: queijo de minas frescal adicionado de Lactobacillus acidophilus; T3: queijo de minas frescal adicionado de ácido lático.

Os valores de acidez titulável no $12^{\circ}$ dia de fabricação em T2 e T3 foram aproximados. Porém, a acidez no T3 foi maior do que o T2 no $30^{\circ}$ dia de fabricação. Lourenço Neto (1998) observou que a acidez titulável dos queijos com a adição de culturas probióticas (L. acidophilus, Bifidobacterium bifidus e Streptococcus thermophilus) foi maior do que com a adição de ácido lático, sendo os valores, respectivamente, em torno de 0,60 e 0,45g de ácido lático/100g no $21^{\circ}$ dia de armazenagem. A incorporação de outros microrganismos, inclusive o $\mathrm{S}$. thermophilus, que é um rápido acidificante, além do $L$. acidophilus, influenciou o aumento da acidez.

Em T1 e T2 ocorreu aumento semelhante na acidez titulável, ao longo do tempo de estocagem. Resultados semelhantes foram encontrados por Souza (2006), que avaliou a acidez titulável do queijo de minas frescal adicionado de L. acidophilus e no tratamento tradicional e constatou que ocorreu aumento significativo da acidez ao longo de 21 dias de armazenagem para os dois tratamentos, porém não ocorreu diferença significativa entre os dois tratamentos. Os valores encontrados por Souza (2006) foram menores que no presente estudo, sendo no primeiro dia e no $21^{\circ}$ dia de armazenagem, respectivamente, $0,17 \mathrm{~g}$ e $0,28 \mathrm{~g}$ de ácido lático $/ 100 \mathrm{~g}$ no tratamento com $L$. acidophilus e $0,18 \mathrm{~g}$ e 0,29g de ácido lático/100g no tratamento tradicional.

Os resultados das análises de $\mathrm{pH}$, durante a armazenagem, nas amostras de queijo de minas frescal, encontram-se na Fig. 2. O T3, com um dia de fabricação, apresentou valores semelhantes aos encontrados na literatura. Campos e Viotto (1999), Furtado e Lourenço Neto (1994) e Lourenço Neto (1998) obtiveram resultados de $\mathrm{pH}$ entre 6,1 e 6,76.

A diferença do $\mathrm{pH}$ entre $\mathrm{T} 1$ e $\mathrm{T} 2$ no final da armazenagem foi de 0,01, respectivamente, 6,74 e 6,73. Estes resultados diferem dos encontrados por Souza (2006), ao verificar que, no queijo tradicional, não ocorreu redução significativa no $\mathrm{pH}$ ao longo da armazenagem, porém, no queijo adicionado de probiótico, houve redução significativa do $\mathrm{pH}$, em razão da produção de ácidos, principalmente ácido lático, ao longo da armazenagem. Entretanto, Cichoski et al. (2008) quantificaram o pH do queijo prato padrão e do queijo prato com adição de L. rhamnosus, sem adição de cultura lática starter, e determinaram que os valores do $\mathrm{pH}$ encontrados no queijo probiótico foram maiores, em todos os dias analisados, com tendência a aumento do $\mathrm{pH}$ ao longo do tempo de estocagem, e significativamente diferentes do queijo padrão. 


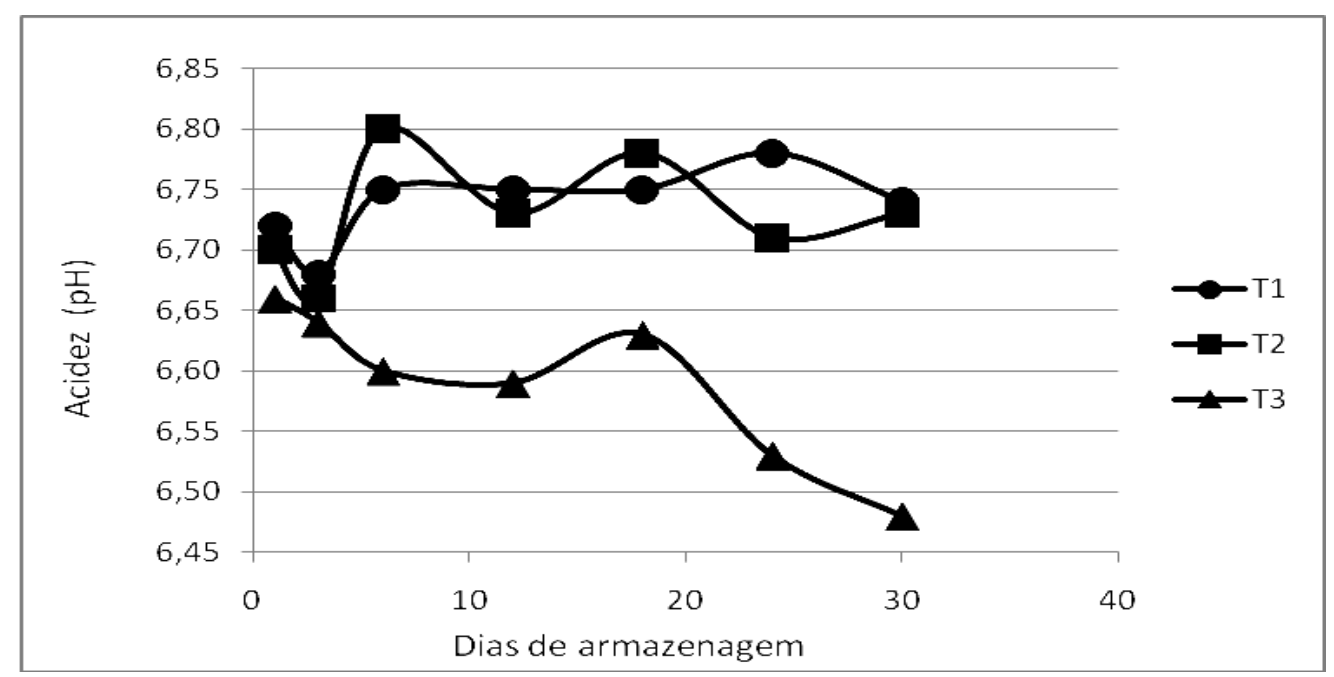

Figura 2. Valores de $\mathrm{pH}$, ao longo do tempo de estocagem dos queijos, segundo os procedimentos de elaboração. T1: queijo de minas frescal tradicional (controle); T2: queijo de minas frescal adicionado de Lactobacillus acidophilus; T3: queijo de minas frescal adicionado de ácido lático.

Em T3 ocorreu a maior redução de $\mathrm{pH}$, sendo de 6,48 com 30 dias de fabricação. Este resultado foi diferente do encontrado por Lourenço Neto (1998), que observou valores mais baixos de $\mathrm{pH}$ no queijo de minas frescal com adição de cultura composta de L. acidophilus, Bifidobacterium bifidus e Streptococcus thermophilus, quando comparado ao queijo adicionado de ácido lático. Os valores encontrados com 21 dias de produção foram 5,82 (com ácido lático) e 5,56 (com a cultura probiótica). Lourenço Neto (1998) justificou que a redução de $\mathrm{pH}$ pode ser atribuída à produção de ácidos pelas bactérias probióticas que compõem a cultura. Porém, Van Dender et al. (1999), ao analisarem o queijo de minas frescal obtido pela adição de ácido lático e por fabricação tradicional, verificaram que não ocorreu mudança significativa no $\mathrm{pH}$ durante a armazenagem por 14 dias. Esses autores encontraram valores de $\mathrm{pH}$ menores que no atual estudo, no $14^{\circ}$ dia, cujo $\mathrm{pH}$ foi de 4,80 nos dois tratamentos.

A adição do L. acidophilus não alterou o pH durante o armazenamento dos queijos no atual experimento, sendo o $\mathrm{pH}$ com um dia de fabricação de 6,70, e com 30 dias de 6,73. Entretanto, estes resultados foram distintos dos encontrados por Buriti et al. (2005) e Ribeiro et al. (2009), que obtiveram redução significativa no $\mathrm{pH}$ dos queijos produzidos como esse probiótico.

A contagem de L. acidophilus nas amostras do T2 encontra-se na Fig. 3. Observa-se que a contagem de L. acidophilus permaneceu alta durante todo o período de armazenagem. Estes resultados assemelham-se aos encontrados por Buriti et al. (2005), que realizaram a contagem de L. acidophilus durante a armazenagem por 21 dias no queijo de minas frescal e não observaram diferença significativa na contagem, pois a bactéria manteve-se viável e constante durante a armazenagem. O mesmo foi verificado por Ribeiro et al. (2009), que estudaram a sobrevivência do L. acidophilus adicionada nas concentrações $10^{6}, 10^{7}, 10^{8} \mathrm{UFC} / \mathrm{g}$ no queijo de minas frescal e verificaram que o decréscimo da população da bactéria probiótica, ao longo do tempo, não foi significativo.

Há uma recomendação para que a quantidade mínima viável diária dos microrganismos probióticos permaneça entre $10^{8}$ e $10^{9} \mathrm{UFC} / \mathrm{mL}$, para que o produto esteja apto para o consumo (Alimentos..., 2008). O T2, a partir da adição inicial de $10^{8} \mathrm{UFC} / \mathrm{g}$, foi eficiente para manter a contagem de microrganismos acima desta quantidade, caracterizando o queijo de minas frescal como alimento probiótico, ao longo de todo o período de armazenagem. 


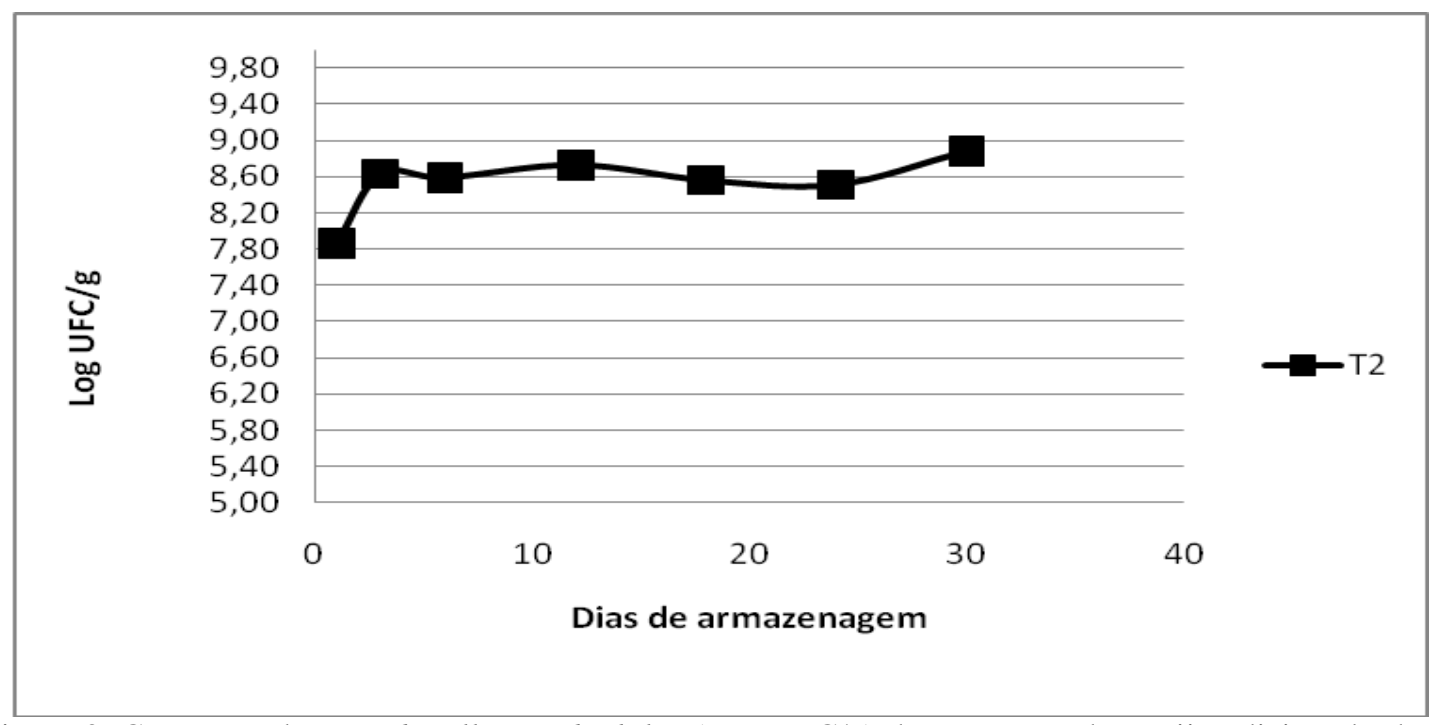

Figura 3. Contagem de Lactobacillus acidophilus (Log UFC/g) das amostras do queijo adicionado de $L$. acidophilus, ao longo do tempo de estocagem.

Os resultados das análises sensoriais do queijo de minas frescal, no sétimo dia de fabricação, pelo teste de aceitação, segundo a escala hedônica de nove pontos, e teste de aceitação, segundo a escala de atitude de nove pontos, encontram-se na Tab. 2.

Tabela 2. Resultados da análise sensorial dos queijos pelo teste de aceitação, segundo a escala hedônica de nove pontos, e pelo teste de atitude, segundo a escala de nove pontos, de acordo com os procedimentos de fabricação

\begin{tabular}{ccc}
\hline Tratamento & $\begin{array}{c}\text { Teste de } \\
\text { aceitação } \\
\text { (médias) }\end{array}$ & $\begin{array}{c}\text { Teste de atitude } \\
\text { (médias) }\end{array}$ \\
\hline T1 & $7,0 \mathrm{~b}$ & $6,6 \mathrm{ab}$ \\
T2 & $7,8 \mathrm{a}$ & $7,1 \mathrm{a}$ \\
T3 & $6,4 \mathrm{~b}$ & $6,0 \mathrm{~b}$ \\
\hline
\end{tabular}

Letras diferentes na coluna indicam que há diferença, pelo teste de Tukey, entre os tratamentos $(\mathrm{P}<0,05)$. T1: queijo de minas frescal tradicional (controle); T2: queijo de minas frescal adicionado de Lactobacillus acidophilus; T3: queijo de minas frescal adicionado de ácido lático.

No teste de aceitação, T2 diferiu significativamente dos demais tratamentos, sendo o mais aceito pelos julgadores, com termo hedônico entre gostei muito e gostei moderadamente. T1 e T3 não diferiram $(\mathrm{P}>0,05)$ entre si. No teste de atitude, T2 não diferiu do $\mathrm{T} 1$, sendo os mais aceitos pelos julgadores, com termo hedônico entre comeria muito frequentemente e comeria frequentemente (T2) e comeria frequentemente e gostei e comeria de vez em quando (T1). O T3 foi o menos aceito entre os julgadores, com o termo hedônico gostei e comeria de vez em quando.

Nas análises sensoriais, foi verificado que a adição do L. acidophilus aumentou a qualidade sensorial do queijo de minas frescal. Outros pesquisadores também verificaram melhoras sensoriais com a incorporação de L. acidophilus, como Buriti et al. (2005), que avaliaram sensorialmente os queijos e concluíram que o queijo contendo o probiótico foi o preferido pelos consumidores, descrito no estudo como o mais agradável. Entretanto, não foi detectada diferença significativa entre os queijos. Souza (2006) avaliou as características do queijo de minas frescal elaborado L. acidophilus e também verificou que a adição do microrganismo probiótico resultou em benefícios sensoriais ao produto. Os benefícios da incorporação de $L$. acidophilus foram maiores que os dos queijos produzidos somente com acidificação direta do leite com ácido lático. Os queijos produzidos com ácido lático, no atual experimento, também não acrescentaram melhoras sensoriais ao produto.

As características sensoriais desempenharam papel importante na aceitação dos alimentos pelos consumidores. A melhor aceitação das amostras de queijo do tratamento com a adição 
do L. acidophilus pode ser atribuída à produção de diacetil e acetaldeído pelo L. acidophilus, conforme mencionaram Oliveira et al. (2002).

\section{CONCLUSÕES}

O L. acidophilus não alterou as características físico-químicas do queijo, por isso pode ser adicionado, sem prejuízos, à qualidade do produto. $\mathrm{O}$ queijo com a adição de ácido lático apresenta teor de gordura mais alto, e as demais características de composição não se alteram durante os 30 dias de armazenagem. O queijo de minas frescal é adequado para a incorporação da bactéria probiótica L acidophilus, pois a cultura empregada permanece em níveis altos durante todo o período de armazenagem, apresentando populações suficientes para caracterizá-lo como potencialmente probiótico. Nos testes afetivos empregados nas análises sensoriais, o $L$. acidophilus aumenta a qualidade sensorial do queijo de minas frescal, sendo o preferido pelos julgadores. O tratamento composto de ácido lático é o de pior qualidade sensorial, quando comparado aos demais tratamentos testados.

\section{REFERÊNCIAS BIBLIOGRÁFICAS}

ALIMENTOS com alegação de propriedades funcionais e ou de saúde, novos alimentos/ingredientes, substâncias bioativas e probióticos, 2008. Disponível em: <http://www.anvisa.gov.br/alimentos/comissoes/ tecno_lista>. Acessado em: 25/08/2009.

BARRETO, G.P.M.; SILVA, N.; SILVA, E.N. et al . Quantificação de Lactobacillus acidophilus, bifidobactérias e bactérias totais em produtos probióticos comercializados no Brasil. Braz. J. Food Technol., v.6, p.119-126, 2003.

BRASIL. Ministério da Agricultura, Pecuária e Abastecimento. Instrução Normativa ${ }^{\circ}$ 68, de 12 de dezembro de 2006. Oficializa os métodos analíticos oficiais físico-químicos para controle de leite e produtos lácteos. Diário Oficial da União, Brasília, DF, 14 dez. 2006.

BRASIL. Ministério da Agricultura, Pecuária e Abastecimento. Secretaria Nacional de Inspeção de Produtos de Origem Animal. Portaria n 146, de 07 de março de 1996. Regulamentos Técnicos de Identidade e Qualidade dos Produtos Lácteos. Diário Oficial da União, Brasília, DF, 1996.
BURITI, F.C.A.; ROCHA, J.S.; SAAD, S.M. I. Incorporation of Lactobacillus acidophilus in Minas fresh cheese and its implications for textural and sensorial properties during storage. Int. Dairy J., v. 15, p.1279-1288, 2005.

CAMPOS, A.C.; VIOTTO, W.H. Rendimento do queijo minas frescal fabricado com ácido lático e diferentes proporções de fermento lático. In: CONGRESSO NACIONAL DE LATICÍNIOS, 16., Juiz de Fora. Anais..., Juiz de Fora: Instituto de Laticínios Cândido Tostes, 1999. v. 54, p. 105-109.

CICHOSKI, A.J.; CUNICO, C.; DI LUCCIO, M. et al. Efeito da adição de probióticos sobre as características de queijo prato com reduzido teor de gordura fabricado com fibras e lactato de potássio. Cienc. Tecnol. Aliment., v.28, p.214219, 2008.

ESTATÍSTICAS do leite. Juiz de Fora: Embrapa/CNPGL, 2010. Disponível em: <http://www.cnpgl.embrapa.br/>. Acessado em: 5 jan. 2010.

FOX, P.F.; GUINEE, T.P.; COGAN, T.M.; SWEENEY, P.L.H. Fundamentals of cheese science. Maryland: Aspen Publication, 2000. $587 \mathrm{p}$.

FURTADO, M.M.; LOURENÇO NETO, J.P.M. Tecnologia de queijos: manual técnico para a produção industrial de queijos. São Paulo: Dipemar, 1994. 118 p.

GARCIA, G.R.; SCHOCKEN-ITURRINO, R.P.; MEDEIROS, A.P. et al. Inibição do crescimento de bactérias patogênicas por Lactobacillus acidophilus. Rev. Port. Cienc. Vet., v.101, p.263268, 2006.

LOURENÇO NETO, J.P.M. Uso de culturas láticas na fabricação de Minas Frescal com alternativa de melhoria de qualidade. In: SEMINÁRIO INTERNACIONAL QUEIJOS FRESCOS, 1., Atibaia, SP, 1998. p.59-75.

MÉTODOS analíticos oficiais para controle de produtos de origem animal e seus ingredientes. Brasília, DF: LANARA, 1981.

MÉTODOS físico-químicos para análise de alimentos. 4.ed. Brasília: Instituto Adolfo Lutz, 2005. 
OLIVEIRA, M.N.; SIVIERI, K.; ALEGRO, J.H.A. et al. Aspectos tecnológicos de alimentos funcionais contendo probióticos. Braz. J. Pharm. Sci., v.38, p.1-21, 2002.

PERRY, K.S.P. Queijos: aspectos químicos, bioquímicos e microbiológicos. Quim. Nova, v.27, p.293-300, 2004.

PINTO, C.P.; MASCARENHAS, M.O.; FIGUEIREDO, H.M. et al. Queijo Minas Frescal: avaliação da qualidade microbiológica. In: CONGRESSO NACIONAL DE LATICÍNIOS, 21., Juiz de Fora. Anais..., Juiz de Fora: Instituto de Laticínios Cândido Tostes, 2004. v.59, p.198-201.

RIBEIRO, E.P.; SIMÕES, L.G.; JURKIEWICZ, C.H. Desenvolvimento de queijo minas frescal adicionado de Lactobacillus acidophilus produzido a partir de retentados de ultrafiltração. Cienc. Tecnol. Aliment., v.29, p.19-23, 2009.
SOUZA, C.H.B. Influência de uma cultura 'starter' termofílica sobre a viabilidade de "Lactobacillus acidophilus" e as características de queijo minas frescal probiótico. São Paulo. 2006. 110f. Dissertação (Mestrado em Tecnologia Bioquímico-Farmaceutica) Faculdade de Ciências Farmacêuticas, Universidade de São Paulo, São Paulo, SP.

VAN DENDER, A.G.F.; MASSAGUER-ROIG, S.; CAMPOS, S.D.S. Alterações físico-químicas e vida-de-prateleira do queijo Minas frescal tradicional e fabricado pelo método MMV. In: CONGRESSO NACIONAL DE LATICÍNIOS, 16., Juiz de Fora. Anais..., Juiz de Fora: Instituto de Laticínios Cândido Tostes, 1999. v.54, p. 6782 . 